\title{
1-S06-1 Symposium6
}

\section{Neuroprotective properties of the excitatory amino acid carrier 1 (EAAC1).}

\section{$\underline{\text { Koji Aoyama }}$}

Dept. Pharmacol.,Sch. Med. Teikyo Univ.

Glutathione (GSH) is produced intracellularly from glutamate, cysteine, and glycine. In neurons, cysteine $\bigotimes \bigotimes$ uptake is the rate-limiting step of GSH production mediated by excitatory amino acid carrier 1 (EAAC1). The dysfunction of EAAC1 reduces intracellular cysteine $\bigotimes$ 冈uptake and suppresses neuronal GSH production, causing neurodegeneration. EAAC1 dysfuction has been reported in the brain of patients with neurodegenerative diseases, suggesting that promoting the function of EAAC1 may lead to suppression of neurodegeneration.

Our studies have suggested that both glutamate transporter-associated protein 3-18 (GTRAP3-18), which is an endoplasmic reticulum protein, and miR-96-5p, which is one of microRNAs, are involved in the regulation of EAAC1 functions. Since GTRAP3-18 directly binds to EAAC1 to suppress the expression of EAAC1 on the cell membrane, suppression of GTRAP3-18 promotes the function of EAAC1. MiR-96-5p acts directly on the 3'-UTR of EAAC1 mRNA and suppresses the expression of EAAC1 protein. Recent results of our studies indicate that treatment of antimiR-96-5p can promote the function of EAAC1 to increase neuronal GSH levels in vitro and in vivo.

In this symposium, we will focus on neuroprotective effects of EAAC1 by regulating GTRAP3-18 or miR-96-5p. 